\title{
Acute Osteomyelitis of the Mandible by Extended-Spectrum $\beta$-Lactamase Producing Klebsiella Pneumoniae: A Case Report
}

\author{
Gyeo-Woon Jung, Seong-Yong Moon, Ji-Su Oh, Hae-In Choi, Jae-Seek You
}

\author{
Department of Oral and Maxillofacial Surgery, School of Dentistry, Chosun University, Gwangju, Korea
}

Received June 25, 2021

Revised August 24, 2021

Accepted August 25, 2021
Acute osteomyelitis caused by Klebsiella pneumoniae is rare in the oral and maxillofacial region. Klebsiella pneumoniae is a Gram-negative bacillus and the normal flora of the human body, but it can cause pneumonia, urinary tract infection, meningitis, and osteomyelitis in patient with compromised immune systems. These infections are mainly caused by nosocomial infection. Microbacterial osteomyelitis was developed by clinical cause such as tooth extraction, fracture, and surgical history, which requires long-term antibiotic administration and surgical treatment. This report describes that a 56-year-old male patient with acute osteomyelitis caused by Klebsiella pneumoniae infection after implant placement was treated with intravenous administration of ertapenem without open surgery treatment. Through this case, we report that antibiotic susceptibility test is essential for the treatment of acute osteomyelitis caused by a bacterial infection resistant to empirical antibiotics, and early administration of appropriate antibiotics can reduce the possibility of extensive bone destruction or additional open surgery.

Key Words: Antibiotics, Bacterial infection, Drug resistance, Klebsiella, Osteomyelitis

\section{INTRODUCTION}

Osteomyelitis of the jaw is a relatively rare infectious disease caused by systemic diseases such as hematogenous, bone disease or vascular disease, and odontogenic or nonodontogenic local infection in the oral and maxillofacial region. Osteomyelitis can be classified into two types: acute suppurative osteomyelitis and chronic suppurative osteomyelitis. Acute suppurative osteomyelitis develops shortly after the predisposition occurs and is accompanied by systemic symptoms such as severe pain, tenderness, swelling, fever, and malaise. This osteomyelitis is not completely cured even with appropriate treatment and lasts for 1 to 4 months or longer. And it can become fixed as chronic suppurative osteomyelitis and require the treatments containing sequestrectomy, saucerization, hyperbaric oxygen therapy, mandibular resection and reconstruction [1]. Today, as nutritional status is better and interest in medical care is increasing compared to the past, more patients are visiting hospitals early, and osteomyelitis of the jaw, excluding drug-related osteonecrosis, is gradually decreasing [2]. However, it is difficult to diagnose the acute osteomyelitis quickly and to administer susceptible antibiotics because the bone changes do not appear early on the radiograph. Therefore, acute osteomyelitis is still considered a refractory disease [3].

Enterobacteriaceae producing extended-spectrum $\beta$-lactamase (ESBL) have recently been reported as nosocomial or community infection organism, and ESBL-positive Escherichia coli (E. coli) and Klebsiella pneumoniae are representative [4].

This case reports that acute osteomyelitis with a rapid destruction of the mandible caused by ESBL-producing Klebsiella pneumoniae in a patient with diabetes was 
treated through antibiotics without surgery.

\section{CASE REPORT}

Before reporting a case, the conduct with the approval of the Institutional Review Board (IRB) of the Chosun University Dental Hospital (CUDHIRB 2102001 Q) was processed.

A 56-year-old male visited the Department of Oral and Maxillofacial Surgery, Chosun University Dental Hospital and complained of swelling and pain in the right mandibular molar area. The implants of mandibular 2nd premolar, 1st molar, and 2nd molar were placed 1 month before visit, and were removed 3 days before due to consistent pain. But the pain did not disappear, and mild swelling and fever endured.

The patient was diagnosed with diabetes 2 years before and had taken oral hypoglycemic drugs. The glycemic control was good and there were no other underlying diseases. There was no history of osteoporosis medication or injection and radiation exposure.

On panoramic radiograph of the first medication examination, irregular bone erosion and bone loss of left mandible, the site of implant removal, were observed. But no apparent bone destruction was observed (Fig. 1). There was no distinct symptom excluding nonspecific pain, so anti-biotic, amoxicillin-clavulanic acid (Augmentin; Ilsung Pharma Co., Seoul, Korea), and anti-inflammatory analgesic drug were prescribed.

Two days later, the patient visited the emergency room due to severe swelling of submental and submandibular area, trismus, fever, and chills. Vital signs were as follows; blood pressure 130/80 mmHg, pulse rate 80 beats/min, breath rate 20 times/min, body temperature 36 degrees. In peripheral blood examination, the white blood cells (WBCs) were $12,000 / \mathrm{mm}^{3}$ and the fasting blood glucose was 250 $\mathrm{mg} / \mathrm{dL}$. Enhanced neck CT was taken, and abscess was observed in the submental space (Fig. 2). Under local anesthesia, incision and drainage were performed with pus culture for the antibiotic susceptibility test. Amoxicillin-clavulanic acid (Amocla inj.; Kuhnil Pharma Co., Seoul, Korea) was administered intravenously three times a day at a dose of 1.2 $\mathrm{g}$ for a week and dressing on abscess site was done daily with saline irrigation. However, the amount of pus drained was not reduced and severe pain was remained. The symptoms were not subsided for a week and antibiotic resistance was suspected. ESBL producing Klebsiella pneumoniae was



Fig. 1. Panoramic radiographic image (arrow) on first visit was observed irregular bone erosion and bone loss of left mandible, but no apparent bone destruction was observed.
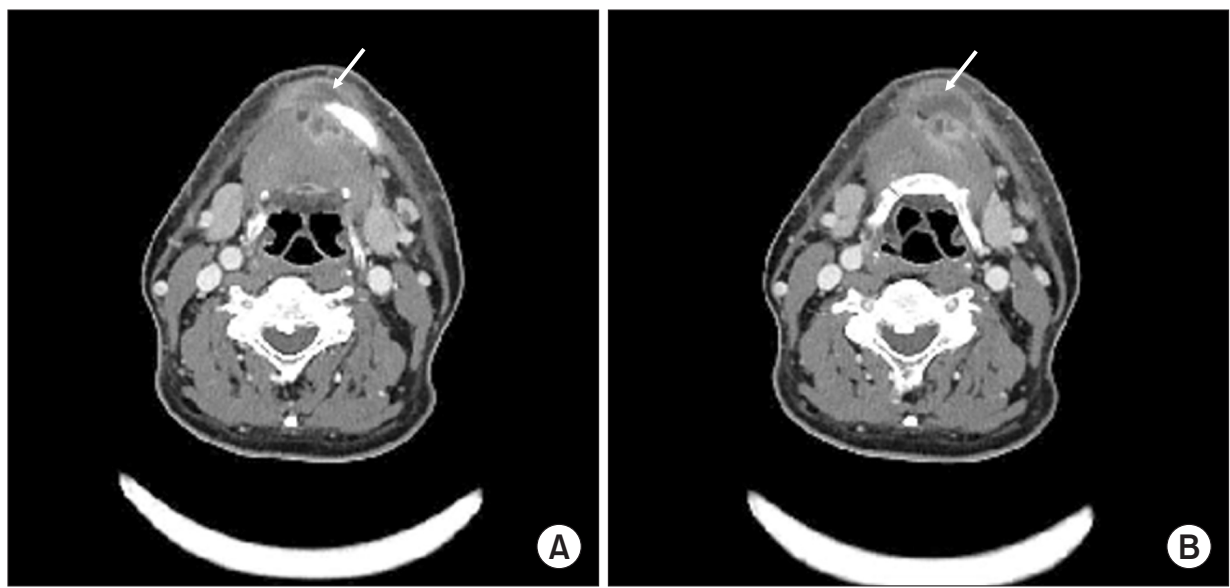

Fig. 2. Radiographic images of enhanced neck computed tomography. (A, B) Axial ct image show abscess (arrow) in the submental space. 
identified in the antibiotic susceptibility test and we consulted with the Infectious Medicine Department. From the 10th day of admission, ertapenem (Invanz Inj.; Merck Sharp \& Dohme Korea Co., Seoul, Korea) was administered intravenously once a day at a dose of $1 \mathrm{~g}$. After 18 days of admission, was not discharged on drainage site, the pain and swelling disappeared. Also, WBCs were $6,000 / \mathrm{mm}^{3}$ in complete blood cell test, all symptoms were improved, and the patient was discharged.

Panoramic radiograph and cone beam computer tomography (CBCT) taken on the day of discharge showed cortical bone destruction of mandible border and severe bone erosion (Fig. 3). Under the diagnosis of acute osteomyelitis, ertapenem (Invanz Inj. $1 \mathrm{~g}$ ) was administered intravenously for 6 weeks and panorama radiograph was taken every 2 weeks to observe the healing pattern. After 4 months of discharge, the cortical bone continuity of mandible border was observed on panoramic radiograph and CBCT (Fig. 4).

\section{DISCUSSION}

Osteomyelitis is an inflammatory condition of the bones by infection through bone marrow cavity [5]. It is not only mainly caused by bacterial, but also caused by various causes such as dental infection, post-extraction complications, and trauma [5].

Clinical symptoms including pain, swelling, fever, delayed healing and fistula, and radiological findings including cortical bone destruction, a mixed pattern of radiolucent and sclerotic changes, and sequestrum are observed in osteomyelitis [6,7].

Acute osteomyelitis has also the same infection signs such as pain, malaise, fever, and cellulitis. However, radiographic changes are not observed in the early stages of acute osteomyelitis, but observed with bone destruction patterns on radiographic image after 10 to 12 days [8,9]. Because of this reason, it is difficult to provide adequate treatment in the early stages of acute osteomyelitis.

Even in this case, there was no evidence of osteomyelitis on the incipient panoramic radiograph, and the patient's clinical symptoms were improved by antibiotic treatment, so acute osteomyelitis could not be suspected and diagnosed. Panoramic radiographs taken at the day of discharge showed partial cortical bone destruction of the mandibular margin, which is to mean the course of healing of acute osteomyelitis.

In the oral and maxillofacial region, Staphylococcus aureus is a representative microorganism causing osteomyelitis, followed by Arachnia, Klebsiella, mycobacterium tuberculosis, and Eikenella spp. [10]. In addition, osteomyelitis
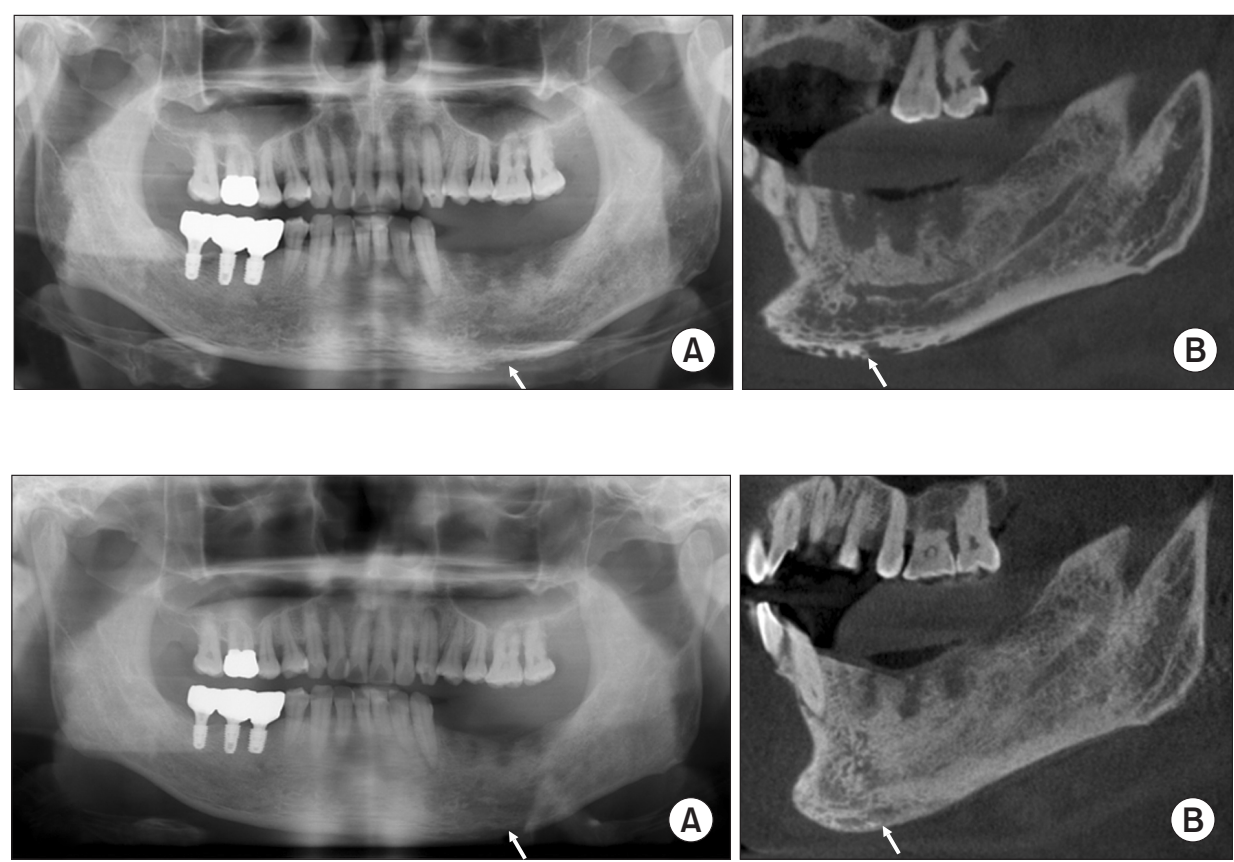

Fig. 4. Radiographic examinations after 4 months of discharge show the cortical bone continuity of mandible border (arrow). (A) Panoramic view. (B) Cone beam computer tomography-sagittal view. 
is caused by fungal organisms such as Aspergillus and Candida parapsilosis, especially in immunocompromised patients [11].

Penicillin is used as the first-choice empiric antibiotic in the oral and maxillofacial region. Intractable microbes can be treated in combination with metronidazole, clindamycin, cephalosporins, amoxicillin/clavulanic acid, carbapenems, vancomycin, and etc [4]. Acute osteomyelitis should be primarily treated with antibiotics. Also, underlying vulnerable conditions must be adequately managed [11,12]. Acute osteomyelitis is usually a polymicrobial infection, so antibiotic susceptibility test should be performed and appropriate antibiotics should be administered intravenously. Clindamycin is an antibiotic used for treatment on streptococci and anaerobes found in acute osteomyelitis. Serious or chronic osteomyelitis requires surgical treatment to remove the infected tissue and necrotic bone [11,12].

Klebsiella pneumoniae identified in this case causes a variety of infections, including pneumonia, liver abscess, urinary tract infections, and bacteremia. Klebsiella pneumoniae mainly causes infections in immunocompromised patients [13]. ESBL enzyme produced by Klebsiella pneumoniae hydrates and inactivates $\beta$-lactams. Therefore, these strains are resistant to penicillin, cephalosporin, aztreonam, and etc. used to empirically primary choice [4]. Among the $\beta$-lactam family of antibiotics, Carbapenems are the effective primary treatment for ESBL-producing bacteria. However, with the increase in the use of carbapenems enterobacteriaceae resistant to carbapenems have emerged, and it make the treatment of multi-resistant bacteria difficult $[14,15]$. Klebsiella pneumoniae carbapenemase producing Klebsiella pneumoniae requires combination therapy with carbapenem and either colistin-polymyxin B or tigecycline [16].

As reported in this case, when acute osteomyelitis occurs through bacterial infection, antibiotics should be administrated intravenously for a long period of time. And osteomyelitis can be treated without open surgery by administering appropriate antibiotics at the exact timing. Although Klebsiella pneumoniae strains are generally resistant to specific antibiotics and carbapenems are effective in many cases, it is not proven that they necessarily follow the tendency. Therefore, the antibiotic susceptibility test is necessary in infection patients, and consultation with the Infectious
Medicine Department is very important to administer the appropriate antibiotic. Also, if surgical intervention is required, it should be performed without delay.

\section{CONFLICT OF INTEREST}

No potential conflict of interest relevant to this article was reported.

\section{ORCID}

\author{
Gyeo-Woon Jung \\ https://orcid.org/0000-0002-1026-0290 \\ Seong-Yong Moon \\ https://orcid.org/0000-0002-7513-4404 \\ $\mathrm{Ji}-\mathrm{Su} \mathrm{Oh}$ \\ https://orcid.org/0000-0002-8369-5025 \\ Hae-In Choi \\ https://orcid.org/0000-0002-2425-9506 \\ Jae-Seek You \\ https://orcid.org/0000-0001-7638-9583
}

\section{REFERENCES}

1. Schuknecht B, Valavanis A. Osteomyelitis of the mandible. Neuroimaging Clin N Am 2003;13:605-618.

2. Hudson JW. Osteomyelitis of the jaws: a 50-year perspective. J Oral Maxillofac Surg 1993;51:1294-1301.

3. Tsujiguchi K, Imai K, Mizuno T. Rapidly progressing osteomyelitis of the mandible: a case report. Jpn J Plast Reconstr Surg 1996;39:641-646.

4. Paterson DL. Recommendation for treatment of severe infections caused by Enterobacteriaceae producing extended-spectrum $\beta$-lactamases (ESBLs). Clin Microbiol Infect 2000;6:460-463.

5. Baur DA, Altay MA, Flores-Hidalgo A, Ort Y, Quereshy FA. Chronic osteomyelitis of the mandible: diagnosis and management--an institution's experience over 7 years. J Oral Maxillofac Surg 2015;73:655-665.

6. Haeffs TH, Scott CA, Campbell TH, Chen Y, August M. Acute and chronic suppurative osteomyelitis of the jaws: a 10-year review and assessment of treatment outcome. J Oral Maxillofac Surg 2018;76:2551-2558.

7. Prasad KC, Prasad SC, Mouli N, Agarwal S. Osteomyelitis in the head and neck. Acta Otolaryngol 2007;127:194-205.

8. Andre CV, Khonsari RH, Ernenwein D, Goudot P, Ruhin B. Osteomyelitis of the jaws: a retrospective series of 40 patients. J Stomatol Oral Maxillofac Surg 2017;118:261-264.

9. Koorbusch GF, Deatherage JR, Curé JK. How can we diagnose 
and treat osteomyelitis of the jaws as early as possible? Oral Maxillofac Surg Clin North Am 2011;23:557-567, vii.

10. Tuon FF, Russo R, Nicodemo AC. Brain abscess secondary to frontal osteomyelitis. Rev Inst Med Trop Sao Paulo 2006;48:233235.

11. Pincus DJ, Armstrong MB, Thaller SR. Osteomyelitis of the craniofacial skeleton. Semin Plast Surg 2009;23:73-79.

12. Dym H, Zeidan J. Microbiology of acute and chronic osteomyelitis and antibiotic treatment. Dent Clin North Am 2017;61:271282.

13. Paczosa MK, Mecsas J. Klebsiella pneumoniae: going on the offense with a strong defense. Microbiol Mol Biol Rev 2016;80:629-
661.

14. Nathisuwan S, Burgess DS, Lewis JS 2nd. Extended-spectrum $\beta$-lactamases: epidemiology, detection, and treatment. Pharmacotherapy 2001;21:920-928.

15. Rupp ME, Fey PD. Extended spectrum $\beta$-lactamase (ESBL)-producing Enterobacteriaceae: considerations for diagnosis, prevention and drug treatment. Drugs 2003;63:353-365.

16. Qureshi ZA, Paterson DL, Potoski BA, et al. Treatment outcome of bacteremia due to KPC-producing Klebsiella pneumoniae: superiority of combination antimicrobial regimens. Antimicrob Agents Chemother 2012;56:2108-2113. 